\title{
Pseudoseptic arthritis: an unusual presentation of neuropathic arthropathy
}

\author{
Worawit Louthrenoo, Barbara E Ostrov, Young S Park, Susan Rothfuss, \\ H Ralph Schumacher Jr
}

\begin{abstract}
A 49 year old black man with scoliosis presented with bilateral shoulder swelling eventually shown to be due to neuropathic arthropathy related to underlying syringomyelia. The synovial fluid was highly inflammatory, but cultures from synovial fluid and synovial tissue were all sterile. Profuse fat droplets were noted and considered as a possible cause of the inflammation. This is an unusual presentation of neuropathic arthropathy in a patient who was also harbouring an adenocarcinoma that was undetected until later.
\end{abstract}

Neuropathic arthropathy is a destructive process which is thought to be caused by impaired articular proprioceptive and pain sensation leading to overuse and repetitive injury and by altered blood flow to the joints. ${ }^{1}$ Synovial fluid is usually 'non-inflammatory' or haemorrhagic. 'We present a patient with syringomyelia who had neuropathic arthropathy of the shoulder and had an unusual highly inflammatory synovial fluid which mimicked septic arthritis.

\section{Case report}

A 49 year old black man presented with bilateral shoulder swelling. Seven months before admission he developed intermittent tingling and numbness in his right hand. Six months later he noted painless swelling of his right shoulder, with weakness and increased numbness in his right arm. Soon after this his left shoulder began to swell, with pain on motion. He denied any history of fever, weight loss, joint stiffness,. back pain, diarrhoea, homosexual activity, intravenous drug abuse, exposure to tuberculosis or syphilis, or trauma to his neck or shoulders. Medical history included hypertension, and scoliosis diagnosed when he was in the army. He had a left total hip arthroplasty in 1982. The cause of his left hip disease was not ascertained. He had gonococcal urethritis on several occasions but had been last treated six months before admission. He admitted past heavy tobacco and alcohol consumption. He also had a history of exposure to asbestos.

Examination showed a pale, alert man with normal vital signs. His back showed marked right thoracic scoliosis with the apex at the T9 vertebral body and a secondary left lumbar scoliosis. His right shoulder was markedly swollen with a massive warm effusion. Range of motion was decreased but was without pain. His left shoulder was moderately swollen, warm, with a large effusion and was painful with motion. Intrinsic muscles of his right hand were atrophied. Muscle strength of both arms seemed decreased but was difficult to assess owing to the mechanical limitation of the shoulders. Biceps and triceps reflexes were absent bilaterally. Pinprick, temperature, and proprioceptive sensations were decreased in both arms, but loss' was more severe on the right. Movement of his left hip was limited without pain. Results of the remainder of the examination were unremarkable.

Initial laboratory tests showed a haemoglobin of $66 \mathrm{~g} / \mathrm{l}$, packed cell volume $0 \cdot 2$, white blood cell count $8 \cdot 9 \times 10^{9} / 1$ with a normal differential count, platelets $1127 \times 10^{9} / 1$, and reticulocyte count $2 \cdot 4 \%$. Erythrocyte sedimentation rate was $150 \mathrm{~mm} / \mathrm{h}$. Peripheral blood smear showed hypochromic and microcytic red cells without other significant abnormalities. Urine analysis results were normal. Stool occult blood was negative.

Chest radiograph showed marked right thoracic scoliosis. The heart and lungs were normal. Cervical spine radiographs showed cervical spondylosis of C3-4 with narrowing of the intervertebral disc spaces. Neuroforamina were normal. Radiographs of his right shoulder showed a large soft tissue density around the shoulder. The joint space was widened. There were destructive changes with resorption of portions of the humeral head and the glenoid (fig 1). Soft tissue calcification, chondro-

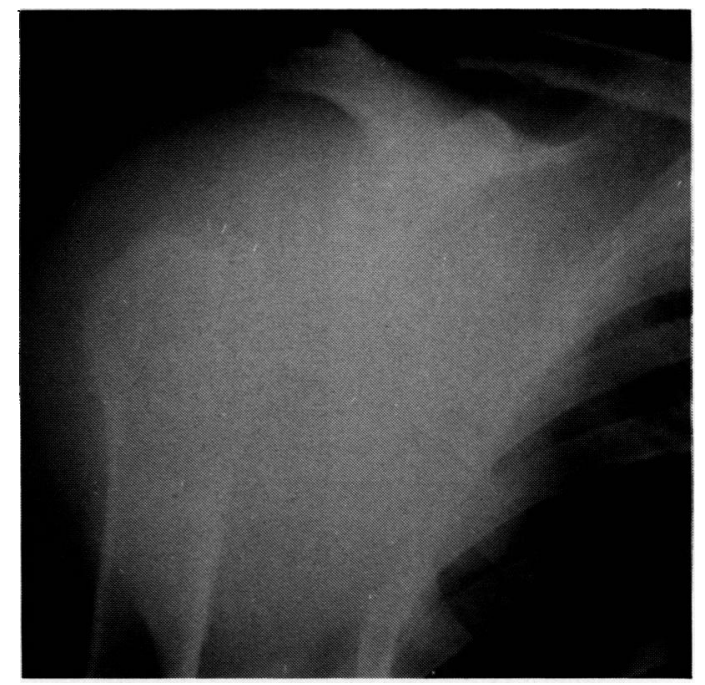

Figure 1 Radiograph of the right shoulder showing a large soft tissue density around the shoulder joint. The joint space is widened and there is lysis of the humeral head and the glenoid surface. No soft tissue calcifications or osteophytes are seen. 
calcinosis, or osteophytes were not identified. Radiographs of his left shoulder showed minimal soft tissue swelling and widening of the glenohumeral joint space. Magnetic resonance imaging of his cervical spine (fig 2) showed a long thin slit in the middle of the upper cervical spinal cord, which is characteristic of collapsed syringomyelia.

Repeated arthrocenteses of his right shoulder obtained $100-500 \mathrm{ml}$ of cloudy serosanguinous synovial fluids. The white blood cell counts ranged between $37 \cdot 3$ and $61 \cdot 1 \times 10^{9} / 1$ with 93-98\% neutrophils. Arthrocentesis of his left shoulder produced $250 \mathrm{ml}$ of serosanguinous fluid. The white blood cell count was $41.6 \times 10^{9} / 1$ with $97 \%$ neutrophils. All fluids contained red

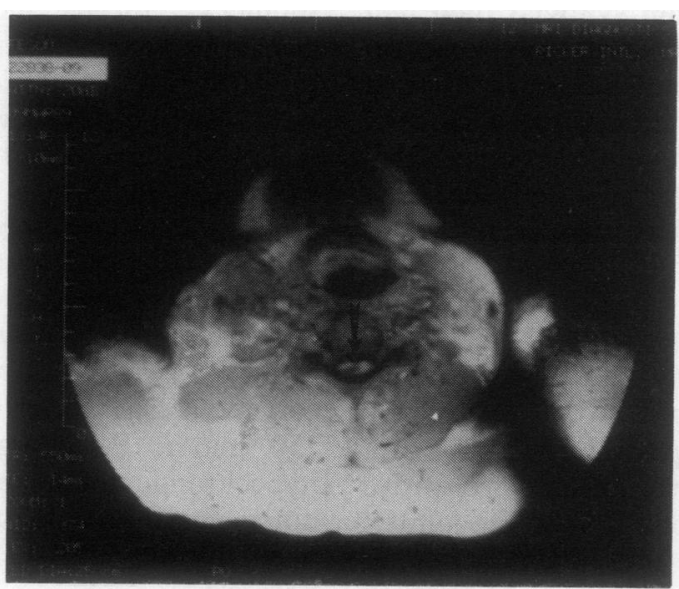

Figure 2 Magnetic resonance imaging of the cervical spine shows a thin transverse slit (arrow) in the middle of the atrophic cervical cord. This is characteristic of a collapsed syringomyelia. cells. There were no monosodium urate or calcium pyrophosphate dihydrate crystals. Alizarin red S staining for calcium crystals was also negative. Numerous intracellular and extracellular fat droplets were identified by Sudan stains. Electron microscopy of the synovial fluid showed degranulated neutrophils containing homogeneous grey globular bodies and other unusual angulated osmiophilic, presumably lipid related, electron dense structures. Closed needle synovial tissue biopsy was performed in his right shoulder (fig $3 A$ ). The synovium was oedematous, had areas with congested vessels, and was infiltrated with neutrophils, lymphocytes, plasma cells, and some necrotic cells, which were scattered throughout the tissue. Extravasation of red blood cells was also noted. Tissues stained for micro-organisms were negative. Cultures for bacteria, fungi, and mycobacteria from synovial fluid and synovial tissue were sterile.

As there was still concern about the possibility of infection an open arthrotomy and synovial biopsy of his right shoulder was performed two weeks later. At surgery the humeral head was found to be grossly deformed with a thick fibrin exudate covering the bone. The cartilage was completely destroyed. There was no synovial fluid obtainable at this time. Microscopically, the synovial surface was covered with fibrin. There was proliferation of the synovial lining cells. The subsynovial cell layer showed proliferation of small blood vessels, prominent fibrous tissue, and some chronic inflammatory cell infiltration. There was no evidence of granulomas or tumour. Fragments of bone and cartilage were seen embedded in the synovium (fig 3B). Smears stained for micro-organisms

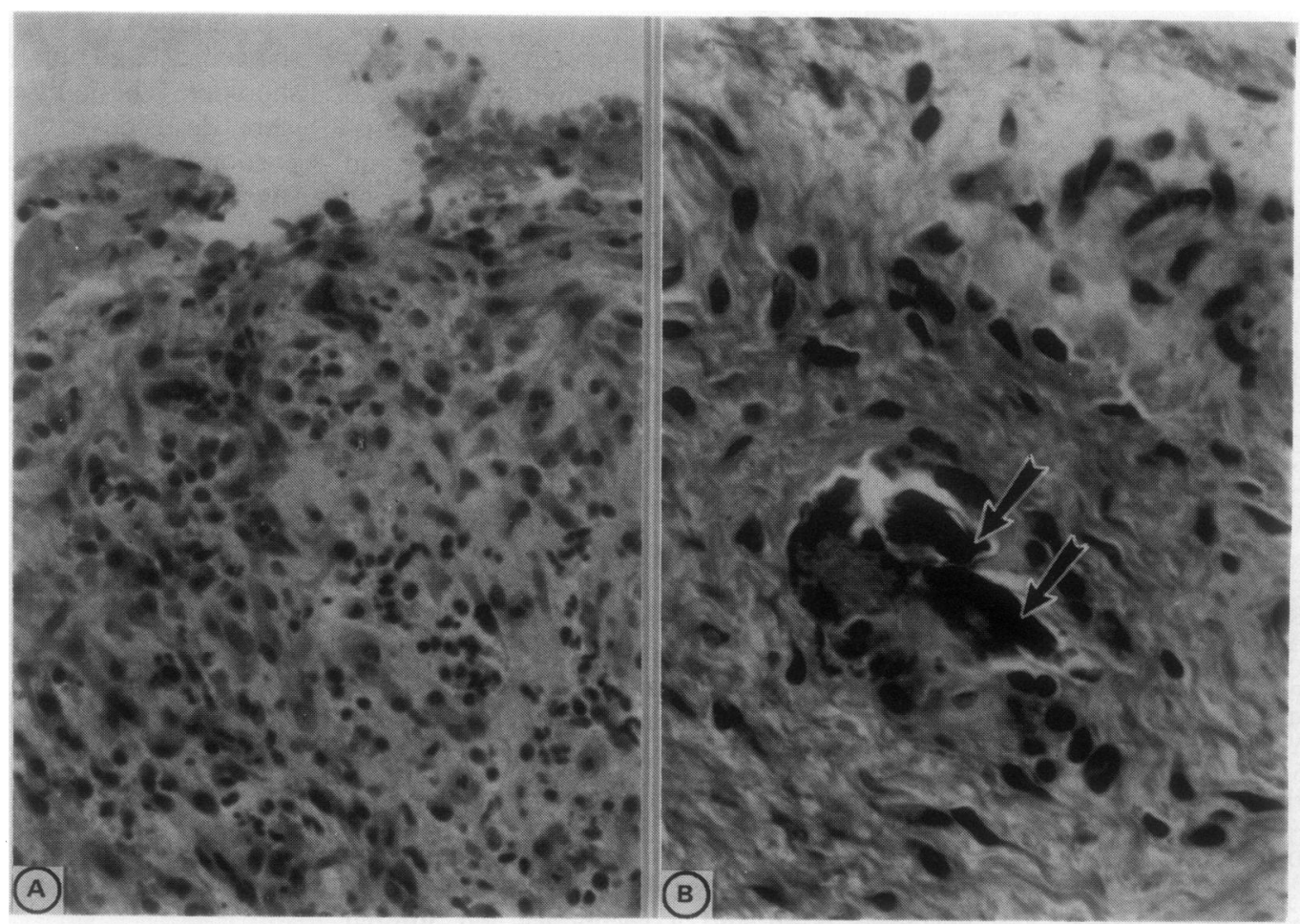

Figure 3 Synovial tissue of the right shoulder. (A) The synovium obtained by needle biopsy after one month of swelling in this area is heavily infiltrated with acute inflammatory cells (haematoxylin and eosin). (B) Two weeks later most areas of the synovium show vessels with thickened walls. Fragments of bone (arrows) are seen in the synovium (haematoxylin and eosin, using green filter). 
were negative as were stains for amyloid. Synoyial tissue cultures for bacteria, mycobacteria, and fungi were all sterile. Electron microscopy of the synovial lining cells showed many lipid droplets and osmiophilic membranous arrays suggestive of phospholipids (fig 4 ). No crystals or any suggestions of viral or bacteria-like particles were seen under electron microscopy.

Blood electrolyte and liver enzyme levels were normal. Serum albumin was $24 \mathrm{~g} / 1$ (normal 35-55) and globulin $66 \mathrm{~g} / 1$ (normal 25-29). Serum protein and immune electrophoresis showed IgG and IgA polyclonal hypergammaglobulinaemia. Results of iron studies were consistent with anaemia of chronic illness. Immunological test results, including antinuclear antibodies, rheumatoid factors, antiSSA, anti-SSB, anti-Sm, anti-RNP, serum and cerebrospinal fluid test for syphilis, purified protein derivative skin tests, Lyme antibody, serum complements, circulating immune complexes, HIV antibody, HLA-B27. and B7, were all normal or negative. Cerebrospinal fluid was normal. Bone marrow biopsy showed a hypercellular marrow with mature erythroid, myeloid, and megakaryocytic series. Plasma cells were normal. Bone marrow iron storage was increased.

He was given non-steroidal anti-inflammatory drugs for the mild left shoulder pain and

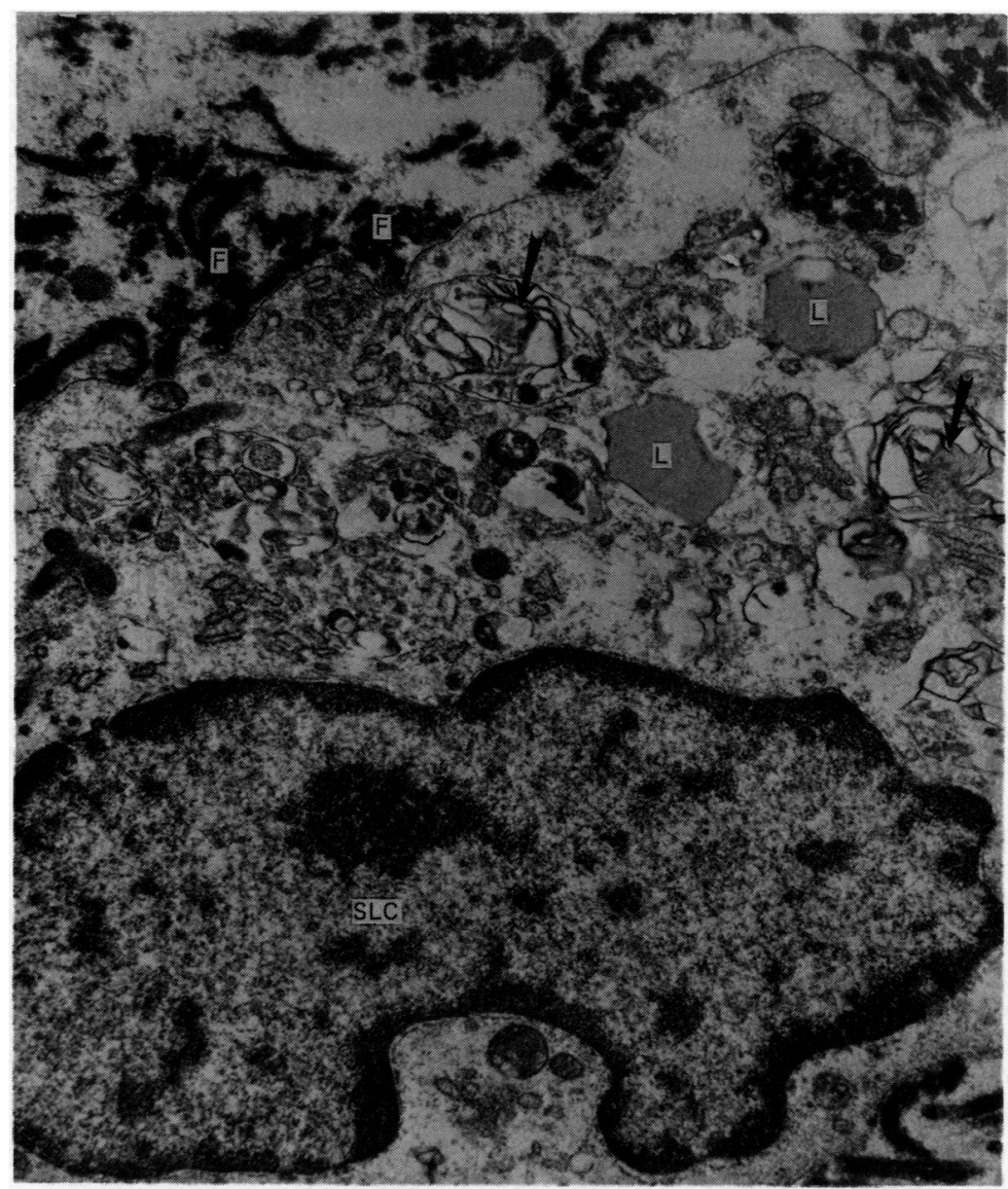

Figure 4 Electron microscopic study of the synovium shows synovial lining cells with homogeneous lipid droplets $(L)$ and membranous arrays of presumed lipids (arrows). $S L C=$ nucleus of synovial lining cells. $F=$ fibrin on the surface. received physical treatment. He was never given antibiotics. Both shoulders showed some functional improvement and were less swollen. After discharge from hospital he was lost to follow up until he was admitted again one year later with chronic cough and progressive shortness of breath. Examination showed diminished breath sounds in his right lung and palpable right supraclavicular and axillary lymph nodes. His right shoulder showed minimal swelling without effusion. There were no changes in neurological findings. A chest radiograph showed diffuse alveolar infiltrates in the lower two thirds of the right lung, thickening of the pleura, and a minimal right pleural effusion. He was hypoxaemic. His haemoglobin had risen to $134 \mathrm{~g} / \mathrm{l}$ and the thrombocytosis had disappeared. He still had an erythrocyte sedimentation rate of $160 \mathrm{~mm} / \mathrm{h}$. His liver panel and acid phosphatase were normal. Radiographs of his right shoulder showed progressive destruction of the humeral head and the glenoid. Pleural fluid study was negative for malignancy. Supraclavicular lymph node biopsy was done for further evaluation of his lung problem and showed metastatic adenocarcinoma thought most likely to have been from the lung. He declined further investigation.

\section{Discussion}

The diagnosis of neuropathic arthropathy is often delayed. Our patient had disease for seven months but had not had any previous evaluation of his painless right shoulder swelling. Various diseases have been associated with neuropathic arthropathy. In addition to syringomyelia found in this case, these include tabes dorsalis, diabetes mellitus, spinal cord and peripheral nerve injury, leprosy, myelomeningocele, amyloidosis, congenital insensitivity to pain, and familial dysautonomia. ${ }^{12}$ Two forms of neuropathic arthropathy have been described-the 'atrophic or resorptive' and the 'hypertrophic or productive' forms. ${ }^{12}$ The former usually affects the non-weightbearing joints-for example, shoulder, elbow, and wrist. These joints develop severe destruction rapidly, sometimes in less than six weeks. ${ }^{3}$ The mechanism is believed to include neurovascular changes in that a loss of neural supply causes alteration in blood-bone flow, leading to active hyperaemia and active bone resorption. ${ }^{2}$ In contrast, the hypertrophic form usually affects the weightbearing joints-for example, hip and knee. In these areas the disease often takes months to years to develop and hypertrophic osteophytes are present. ${ }^{1}$ Impaired sensation may cause overuse and the unprotected joints are subjected to repeated minor injury. The full reasons underlying the development of the two distinct forms or extremes of neuropathic arthropathy are not clear. Norman believed that both types were the same disease but differed in time sequence of manifestation. ${ }^{3}$ Our patient had the atrophic form of neuropathic arthropathy; he developed rapid destruction of his right shoulder over a month and the radiographs showed destruction of the humeral head and the glenoid without hypertrophic osteophytes. The intensely inflammatory synovial fluid noted in our patient 
may be an important factor as is described below.

Neuropathic arthropathy is present in up to one third of patients with syringomyelia. The fingers are affected in up to $80 \%$ of these patients. ${ }^{4}$ Shoulder swelling can be the presenting sign and the shoulder, as in our patient, is the most common joint affected. ${ }^{4} 5$ Scoliosis, most often in the thoracic spine, is common in syringomyelia and is seen in up to $63 \%$ of patients. ${ }^{67}$ Scoliosis can present for many years before the clinical neurological deficits become apparent. ${ }^{7}$ It is not clear whether a shared developmental defect predisposes to both scoliosis and syringomyelia or whether an imbalance of muscle tension results from the neuropathic process in the same case. ${ }^{6}$

The presence of such an inflammatory synovial fluid in our case is of interest. The negative synovial fluid and synovial tissue cultures ruled out septic arthritis as a cause of this patient's joint disease. Synovial fluids in neuropathic joints have been examined in several studies and have generally had leucocyte counts less than $0.65 \times 10^{9} / 1$ but with widely varying percentages of neutrophils $(0-95 \%){ }^{8}$ Occasional higher leucocyte counts up to $19.0 \times 10^{9} / 1$ have been seen in patients who had associated calcium pyrophosphate dihydrate deposition. ${ }^{9}$ Hydroxyapatite crystals also have been reported as a cause of a rapid destructive arthropathy. ${ }^{10} 11$ Neither type of crystal was found in our patient's joint fluid despite the occasional bone fragments in the synovium and bone lysis that might have released bone apatites. Patients specifically identified as having the most rapid resorptive atrophic form of neutropathic joints by radiologists ${ }^{3} 1213$ have not had details of their synovial fluid analysis or histology reported. It might be important to study more of these patients to see if they might have highly inflammatory effusions early in the disease like our patient.

At this time we cannot totally exclude the possibility that the inflammation in the neuropathic joint of our patient was due to a separate process superimposed on the neuropathic arthropathy, such as a seronegative spondyloarthropathy, because he had a history of urethritis. He had no other clues to this, however, was negative for both HLA-B7 and $B 27$, and involvement limited to the joints with neuropathic arthropathy would be most unusual unless somehow the vascular congestion favoured this. A paraneoplastic syndrome was also considered, but all signs of inflammation had subsided even as the tumour progressed.

The presence of fat droplets has not been reported in the synovial fluid or synovial tissue of patients with neuropathic arthropathy. Lipid droplets can come from membranes of degenerated erythrocytes or other cells, or from bone marrow as a result of fracture or other destruction giving the marrow access to the joint space. ${ }^{14}$ Fat droplets or lipid liquid crystals have been shown to be causes of an inflammatory arthritis. ${ }^{15}{ }^{16} \mathrm{We}$ suggest that the neuropathic arthropathy of the shoulder in our patient might have caused chronic bleeding into the destroyed joint space or marrow fat release with phagocytosis of the fat droplets, possibly leading to the inflammatory fluid.

Synovial tissues in neuropathic joints have been reported to show hyperplastic synovium with bone or cartilage debris, or both, and large amounts of haemosiderin in deep macrophages. Chronic inflammatory cell infiltrates with clustered or scattered lymphocytes have been described in some. ${ }^{17} 18$ Subintimal fibrosis has been seen. ${ }^{3}$ Infiltration by neutrophils has not been reported. The first synovial biopsy specimen in our case showed an acute and chronic inflammatory reaction with dramatic polymorphonuclear leucocyte infiltration; two weeks later this was replaced with fibrous and chronic inflammatory cells in the second synovial biopsy specimen. We could not identify bone or cartilage fragments in the first small synovial biopsy specimen but could identify some in the second open synovial biopsy specimen. The presence of severe anaemia and polyclonal hypergammaglobulinaemia in our patient could not be explained by the syrinx and neuropathic arthropathy. The absence of abnormal plasma cells in the bone marrow and the absence of a monoclonal gammaglobulin spike made an underlying plasma cell dyscrasia unlikely. The iron studies were more consistent with the anaemia of chronic disease rather than iron deficiency. The negative amyloid stains in synovial tissue ruled out systemic amyloidosis as a cause of the arthropathy. The subsequently identified adenocarcinoma might have been the cause of these abnormalities and is probably coincidental to the syringomyelia in our case.

In summary, we present the case of a patient with shoulder neuropathic arthropathy and with severe inflammatory synovial fluid. The mechanism for the inflammatory reaction in the synovial fluid is not known, but fat droplets must be considered as a possible cause. Further studies of joint fluids and tissues in patients with the atrophic, resorptive type of neuropathic arthropathy may help us better to understand the mechanisms involved.

1 Ellman M H. Neuropathic joint disease (Charcot joints). In: McCarty D J, ed. Arthritis and allied conditions. 11th ed. Philadelphia: Lea and Febiger, 1989: 1255-72.

2 Brower A C, Allman R M. Pathogenesis of the neuropathic joint: neurotraumatic vs neurovascular. Radiology 1981 139: 349-54.

3 Norman A, Robbins H, Milgram J E. The acute neuropathic arthropathy-a rapid, serverely, disorganizing form of arthritis. Radiology 1968; 90: 1159-64.

4 Skall-Jensen J. Osteoarthropathy in syringomyelia. Analysis of seven cases. Acta Radiol 1952; 38: 382-8.

5 Sackellares J C, Swift T R. Shoulder enlargement as the presenting signs in syringomyelia. Report of two cases and review of the literature. JAMA 1976; 236: 2878-9.

6 Huebert H T, MacKinnon W B. Syringomyelia and scoliosis. f Bone foint Surg [Br] 1969; 51: 338-43.

7 Raininko R. Syringomyelia in scoliotic patients. Amnals of Clinical Research 1986; 18: 93-8.

Ropes $M W$, Bauer $W$. Symovial changes in joint disease. Cambridge: Harvard University Press, 1953: 83-5.

9 Jacobelli S, McCarty D J, Silcox D C, Mall J C. Calcium pyrophosphate dihydrate deposition in neuropathic joints. pyrophosphate dihydrate deposition in neuropathic joints. Four cases of polya

10 Dieppe P A, Doherty M, MacFarlane D G, Hutton C $W$, Brandfield J W, Watt I. Apatite associated destructive Brandfield J W, Watt I. Apatite associate

11 Klimaitis A, Carroll G, Owen E. Rapidly progressive destructive arthropathy of the shoulder-a viewpoint on pathogenesis. I R Reumatol 1988; 15: 1859-62.

$12 \mathrm{Katz}$ I, Rabinowitz J G, Dziadiw R. Early changes in Charcot's joints. American foumal of Roentgenology 1961 86: 965-74. 
13 Meyer G A, Stein J, Poppel M H. Rapid osseous changes in svringomyelia. Radiology $1 \overline{9} \overline{5} \overline{7} ; 6 \overline{9}: 415 \overline{5}-8$.

14 Wise C M, White R E, Agudelo C A. Synovial fluid lipid abnormalities in various disease states: review and classification. Semin Arthritis Rheum 1987; 16: 222-30.

15 Reginato A J, Schumacher H R, Allan D A, Rabinowitz J L. Acute monoarthritis associated with lipid liquid crystals. Ann Rheum Dis 1985; 44: 537-43.

16 Choi S J, Schumacher H R, Clayburne G. Experimental haemarthrosis produces mild inflammation associated with intracellular maltese crosses. Ann Rheum Dis 1986; 45: 1025-8.

17 Horwitz T. Bone and cartilage debris in the synovial membrane. Its significance in the early diagnosis of neuroarthropathy. F Bone foint Surg [Am] 1948; 30: 579-88.

18 Beetham W P, Kaye R L, Polley H F. Charcot's joints. A case of extensive polyarticular involvement and discussion of of extensive polyarticular involvement and discussion of 1963; 58: 1002-12. 